\title{
Ucràina o Ucraìna? Ucràino o ucraìno?
}

\author{
Vittorio Coletti
}

PUBBLICATO: 05 GIUGNO 2015

\begin{abstract}
Quesito:
Sono molti coloro che ci chiedono se si debba dire Ucráina o Ucraina, ucráino o ucraino. In particolare qualcuno ci fa notare che nella scheda sull'accentazione dei nomi geografici consigliavamo Ucráina, ma che, in occasione dei recenti avvenimenti, si sente molto spesso anche la pronuncia Ucraina.
\end{abstract}

\section{Ucràina o Ucraina? Ucráino o ucraino?}

Molti si pongono questa domanda, specie dopo la grave crisi nei rapporti di questo Stato con la Russia. Del resto, non è che uno dei tanti problemi di accentazione dei polisillabi che affliggono o caratterizzano l'italiano di oggi (guaina sarebbe più corretto di guáina e diátriba di diatriba, ma chi usa la forma corretta può facilmente passare per uno che sbaglia!) e che non di rado riguardano proprio i toponimi (Benáco pronunciato Bénaco, Belice pronunciato Bélice). Gli spostamenti dell'accento di parola sono in genere un tipico fattore dell'evoluzione delle lingue (noi diciamo cadére ma i latini dicevano cádere, diciamo ridere e loro dicevano ridére).

Per quanto riguarda i nostri due nomi (e aggettivi) va detto subito che oggi sono accettabili entrambe le pronunce, anche se la più corretta, a rigore, sarebbe quella in passato spesso ritenuta sbagliata, cioè quella con l'accento sulla $i$. Vari dizionari italiani hanno pensato che le due pronunce derivassero dalla diversa accentazione del nome a seconda che fosse pronunciato (con accento sulla $i$ ) in ucraino o (con accento sulla $a$ ) in russo, le due lingue più diffuse in quella regione. In realtà oggi si dice Ucraina, ucraino (Ukraina, ukrainskij) tanto in russo quanto in ucraino. Tuttavia anche in alcuni forum russi attuali si leggono interventi di lettori che chiedono chiarimenti sulla pronuncia, dato che qualche volta succede persino nei loro telegiornali di sentire l'accento sulla a. Il Dizionario degli accenti russi (Slovar' udarenii russkogo jazyka), che ammette come pronuncia corretta dell'aggettivo solo ukrainskij, aggiunge questa precisazione: «l'accentazione ukràjnskij (dall'antiquato Ukràina), che si incontra non solo in poesia, ma anche nella conversazione comune, corrisponde alla prassi antica».

Dunque un'oscillazione non del tutto arbitraria in italiano.

La pronuncia Ukràina, ukräinskij, considerata sbagliata da tutti i dizionari russi a partire dagli anni Cinquanta del Novecento ma ritenuta corretta o unica da quelli piu antichi, ha in effetti illustri precedenti letterari, in particolare in poesia: primo fra tutti quello di Puškin, che nel poema Poltava usa ripetutamente la grafia Yкрайна (Ukrajna), in cui l'uso della $i$ breve (й) esclude di per sé l'accento sulla $i$ e lo sposta sulla $a$. Il compositore Rodion Šcedrin, musicando all'inizio degli anni Cinquanta del Novecento alcuni versi proprio della Poltava ("Ticha ukràjnskaja noč"), seguì naturalmente l'accentazione voluta da Puškin, che quindi continua a essere presente nell'orecchio dei russi.Oltre a Puskkin, (1936) anche Mandelštam, sempre in poesia, pone l'accento sulla $a$. Curioso il caso di Taràs Grigòrevič Šcevčenko, poeta del primo Ottocento, nato in Ucraina, ma vissuto poi in Russia, a San Pietroburgo (I8I4-I86I), che usa, scrivendo in russo, la lezione con l'accento sulla $a$.

L'autorevolezza di queste occorrenze e il fatto che si tratti di poeti che continuano a essere letti e studiati determina ancora oggi oscillazioni di accento in russo, documentate per altro nelle cronache storiche fin dal Seicento e causate anche da un'etimologia molto discussa: la parola ucraina (in cui è controversa persino l'origine della $u$ iniziale, in alcune fonti data infatti come o) contiene secondo alcuni il ceppo krái, limite, bordo, confine, secondo altri il ceppo graničnij, di confine. C'è chi sostiene che il primo a chiamare Ucráina l'Ucraina sia stato il re polacco Stefano Batorij, ma oggi anche in polacco l'accento cade sulla $i$. Bisogna osservare che, prima del Seicento, il termine 'ucraina' non si riferiva specificamente alla regione che noi oggi chiamiamo cosi, ma a qualsiasi territorio di confine. Sul vocabolario di Vladimir Dal' (Tolkovkij slovar' zhibogo velikorusskogo jasyka, I882) si ammette per il toponimo solo l'accento sulla $a$, ma l'unico aggettivo che riporta non è ukrainskij, ma ukrájnyj, sempre col significato di dal'nyj (lontano), pograničnij(sul confine). Invece il recente Fasmer (Max Fasmer, Etimologičeskij slovar' russkogo jazyka, Moskva 2004, vol. 4, lemma Ukraina) accetta solo l'accento sulla $i$, 
ma riporta anche alcune espressioni antiche in cui si usava l'accento sulla a e divide l'etimo di ukrájnyj, che deriverebbe da y krajje, presso il confine, sul bordo, dai posteriori ukrainez (abitante dell'Ucraina) e ukrainskij (relativo all'Ucraina), «inizialmente riferito ai soli abitanti dell'Ucraina orientale, poi diffuso su tutto il territorio (dell'attuale Ucraina)».

Insomma, ce n'è quanto basta per comprendere e tollerare le ragioni dell'oscillazione d'accento nella pronuncia italiana della parola in questione, oscillazione che si riscontra oltretutto anche in qualche altra lingua europea. $\mathrm{Ma}$, a rigore, dato che nell'uso moderno le lingue slave, russo incluso, accreditano ormai come unica pronuncia corretta quella accentata sulla $i$ sarebbe meglio dire Ucraina/ucraino, come del resto consiglia l'autorevole DOP.

* Grazie alla collaborazione della musicologa Elisabetta Fava, studiosa di opera russa.

\section{Cita come:}

Vittorio Coletti, Ucràina o Ucraìna? Ucràino o ucraìno?, "Italiano digitale", 2015, VIII, 2019/1 (gennaio-marzo), pp. 57-58.

DOI: $10.35948 / 2532-9006 / 2019.3085$

Copyright 2015 Accademia della Crusca

Pubblicato con licenza creative commons CC BY-NC-ND 